\title{
A study on the behavior of an underwater explosion bubble near a gid all
}

\author{
Ching-Yu Hsu*, Cho-Chung Liang** and Vo-Phuong Duy** \\ * Department of Marine Mechanical Engineering, ROC Naval Academy, Taiwan. \\ ** Department of Mechanical and Automation Engineering, Da-Yeh University, Taiwan. \\ * Corresponding Author: hsuchingyu@gmail.com
}

Submitted :08/04/2020

Revised :03/11/2020

Accepted :09/11/2020

\begin{abstract}
The dynamic approach to an underwater explosion (UNDEX) is a complex episode that involves shockwave propagation, bubble pulse with high pressure, and water jet impact. This paper proposes linkage of Finite Element Avenue (FEM) and Companion of Eulerian-Lagrangian (CEL) to supply promised data of large deformations and flow simulation of fluid and gas where the bubble interaction is near a stiff wall. To conduct the process, a $7.5 \mathrm{~m} \mathrm{x}$ $9.0 \mathrm{~m}$ Eulerian domain and explosive charges of $10 \mathrm{~g}, 35 \mathrm{~g}$ and $55 \mathrm{~g}$ TNT are built in a free field, respectively. Numerical analysis, as far as a comparison with research from E. Klaseboer, has been given in this study. The important results obtained from the CEL approach imply high expectations. In spite of the fact that this approach is not adequately consistent to totally supplant a live test, it can be utilized as an outline database to anticipate outcomes of managing an UNDEX with a high pressure bubble. The behavioral explosion from an UNDEX bubble near a rigid wall is a prospective contribution in this research. With these results, this technique can be used in further studies to examine UNDEX bubbles in the vicinity of deformable and complex structures.
\end{abstract}

Keywords: Underwater explosion; High-pressure bubble; Toroidal bubble.

\section{INTRODUCTION}

An underwater explosion (UNDEX) typically involves two essential and distinct phenomena. An underwater explosion with a strong pressure bubble can repeatedly undergo expansion and contraction circles. Since the bubble exceeds the normal volume, it can also produce secondary pressure pulses, Cole, R.H., 1948. Klaseboer et al. (2005) conducted experiments using different charge masses of hexocire; the major behaviors of the bubbles in the free area and close to the stiff wall were interpreted, and the response of the deformable plate was also examined. The response of complex structures to a bubble collapse load was reported by Brett et al. (2000). They have demonstrated that the strain of broken bubbles plays a vital role in causing damage. In a subsequent investigation, the interaction of an UNDEX bubble with a nearby cylinder was revealed through high-speed video images. Then, the transient behavior of the bubble near the rigid and free surface was analyzed in detail by Blake et al. (1987). Numerous advanced simulation techniques have been introduced to improve simulation accuracy. Zhang et al. (1998) suggested a guided technique for calculating the free surface of solid angles. The above approach has been successfully integrated into a 3D model and applied parallel to the free surface of the water for the interaction of two bubbles. Another technique that should be cited here is the elastic mesh technique (EMT) of Wang et al. 
(1987). Applying computer software to simulate a shockwave in an UNDEX, Jin Qiankun et al. (2011) used finite element models to analyze how a surface ship impact withstands the UNDEX in ABAQUS. However, for solving the interaction between the bubble and structures, the BEM code often needs to be coupled with an FEM code, and the time step must be tightly controlled during the analysis. Furthermore, this coupling is complex, and it is not as popular as FEM is in commercial code. The present study introduced an application of FEM in simulating the UNDEX bubble. The fluid structure interaction was solved using the coupled Eulerian-Lagrangian (CEL) technique, in which the Eulerian technique allowedisimulating multi-materials (detonation gas, air, and water) in an element, and the structure was simulated using Lagrangian elements. Migration of bubbles affected by gravity was considered, and the main characteristics of the bubble were interpreted during the collapse process. The behavior of a bubble close to vertical and horizontal rigid walls was also examined.

\section{THEORETICAL BACKGROUND}

\section{The Migration of Gas ubble Underwater}

Essentially, the development of high-pressure bubbles continues until the velocity of the liquid that surrounds the bubble vanishes. The gas bubble then begins to contract at a rising rate. The bubble process will show the repetition pattern of expansion and contraction. The gaseous bubble created by the UNDEX can be determined with the equation of maximum bubble radius $\left(\mathrm{R}_{\max }\right.$ ) and time $(\mathrm{T})$ to the maximum of the first pulse (Hans U. Mair, 1995):

$$
\begin{aligned}
& R_{\max }=3.50 \frac{W^{\frac{1}{3}}}{z_{0}^{\frac{1}{3}}} \\
& T=2.11 \frac{W^{\frac{1}{3}}}{Z_{0}^{\frac{5}{6}}}
\end{aligned}
$$

where $\mathrm{D}$ is the depth of the explosive in meters, $\mathrm{W}$ is the equivalent mass of the TNT explosive in $\mathrm{kg}$, and $\mathrm{Z}_{0}=\mathrm{D}+9.8$ is the entire static pressure at the site of the explosive. The vertical migration, $\mathrm{m}$, which is outlined as the distance from the locality of the explosive to the position of the first lowest, is experimentally identified as (Hans U. Mair, 1995):

$m=\left(\frac{12.2}{d+9.8}\right) \cdot W^{1 / 2}$

where dis the initial depth of the explosive in meters and $\mathrm{W}$ is the equivalent mass of the TNT explosive in $\mathrm{kg}$.

\section{NumericalBackground}

The Eulerian-Lagrangian technique has a powerful two-way coupling (Jan Erik Olsen, Paal Skjetne, Sep. 2016). Considering a particle's fluid rotation around an axis parallel to the $\mathrm{z}$ axis, the rotation dimension can be defined as the average angular velocity of two linear infinitesimal elements perpendicular to each other and to the rotation axis. The two line elements can be taken as $\delta \mathrm{x}$ and $\delta \mathrm{y}$ and the particle at $\mathrm{P}(\mathrm{x}, \mathrm{y})$ and have velocity components $\mathrm{u}$ and $\mathrm{v}$ in the $\mathrm{xy}$ plane (David Cherney et al.,2013). The angular velocity of $\delta x$ and $\delta y$ are 


$$
\frac{v+(\partial v / \partial x) \delta x-v}{\partial x}=\frac{\partial v}{\partial x}
$$

$$
-\frac{u+(\partial u / \partial y) \delta y-u}{\partial y}=-\frac{\partial u}{\partial y}
$$

$$
\nabla^{2} \Phi=0
$$

The flow function $\Phi$ is a potential function of Laplace's equation.

For the digital simulation of the interactive phenomenon between a high-pressure bubble and a structure, the acoustic structural coupling method from the ABAQUS was applied. This strong, easy-to-use functionality of ABAQUS/Explicit General Contact allows multi-physics simulations, such as interaction between fluid structures, to be completely coupled(ABAQUS Analysis User's Manual, USA, 2011).

\section{NUMERICAL SIMULATION}

The numerical models in this study were developed by replicating the experiments by Klaseboer et al. (2005), which studied the dynamic process of an UNDEX bubble in a pond. Different charge weights of hexocire $(55 \mathrm{~g}, 35 \mathrm{~g}$, and $10 \mathrm{~g}$ ) were positioned at a depth of $3.5 \mathrm{~m}$ located approximately in the middle of the pond. The sidewalls of the pond were designed to diffract the shockwaves of the blast and absorb the strength of the shock explosion. A high-speed camera above the water surface captured the images of the bubble explosion phenomenon. The water and the structural strain were tested using tourmaline piezoelectric sensors.

\section{Finite Element Model}

Bubbles generated by underwater explosions, assuming initially spherical high-pressure bubbles. Moreover, the detonation gas product was simulated as an ideal gas, and the water was assumed to be unobserved. Because the numerical model involved liquid and gas flow that used a Lagrangian standard-based FEM, it was illogical. Therefore, numerical analysis was carried out using a finite volume system in ABAQUS software based on Euler, which enabled the management of too much deformation of the fluid and allowed the simulation of multi-materials. The numerical model contained three regions of material: water, air, and highly compressed detonation gas. As the hydrostatic pressure in the water surrounding the bubble plays an important role in the dynamic bubble phase, the height of the water region in the experimental pond $(7 \mathrm{~m})$ has been retained and the gas bubble has been introduced in the same place as the experimental load $(3.5 \mathrm{~m})$. An air region was created in the model to control the increase of the water surface as the bubble expanded, and to make the simulation more natural. In order to prevent materials from flowing out, all faces of the Eulerian domain were set, and a non-reflective Eulerian boundary was added to the faces of the Eulerian domain to remove the reflection of the pressure waves that would have influenced the accuracy of the simulation tests. 


\section{Initial Conditions}

The air pressure was equal to the pressure of the atmosphere. The original hydrostatic pressure in the water was the same as the air at the ground point between the regions of air and water, which increased as a result of the water depth rising. The bubble's initial conditions were calculated on the basis of Cole's data [1]. If the charge is a sphere, the radius of the charge is determined using

$$
r_{c h}=\left(\frac{3}{4} \frac{W}{\pi \rho_{0}}\right)^{\frac{1}{3}}
$$

where $W$ is the weight $(\mathrm{kg})$ and $\rho_{0}$ is the density of charge.

The pressures are much higher at smaller specific volumes, which corresponds to the increased effect on the internal energy of repulsive forces. The detonation gas product can be considered an ideal gas with a specific heat ratio of 1.25 if the specific volume is sufficiently large. Thus, the initial volume of the bubble became eight times greater, and the density of the bubble became $1 / 8$ of the density of the charge, $\rho_{\mathrm{g}}=\rho_{0} / 8=203.75 \mathrm{~kg} / \mathrm{m}^{3}$.

The specific volume of gas proportional to this density value is

$$
v_{g}=1 / \rho_{\mathrm{g}}=0.00491 \mathrm{~m}^{3} / \mathrm{kg}=4.91 \mathrm{~cm}^{3} / \mathrm{g},
$$

where $P_{g}=5.704107, \mathrm{~Pa}$ is the original bubble pressure equal to this particular volume size. In the proposed method, commercial finite element software ABAQUS 6.11 was used to simulate the dynamic process of a threedimensional underwater explosion bubble. Table 1 shows the physical parameter details of the numerical model.

Table 1.Physical parameters used in the simulation.

\begin{tabular}{|l|l|c|c|c|}
\hline \multicolumn{1}{|c|}{ Material } & \multicolumn{1}{|c|}{ Parameter } & Symbol & Value & Unit \\
\hline \multirow{4}{*}{ Water } & Density & $\rho_{\mathrm{w}}$ & 1000 & $\mathrm{Kg} / \mathrm{m}^{3}$ \\
\cline { 2 - 5 } & Sound speed & $\mathrm{c}_{\mathrm{w}}$ & 1500 & $\mathrm{~m} / \mathrm{s}$ \\
\cline { 2 - 5 } & & & & \\
\hline \multirow{4}{*}{ Air } & Density & $\rho_{\mathrm{a}}$ & 1.17 & $\mathrm{Kg} / \mathrm{m}^{3}$ \\
\cline { 2 - 5 } & Ratio of specific heat & $\gamma_{\mathrm{a}}$ & 1.4 & - \\
\cline { 2 - 5 } & Initial pressure & $\mathrm{P}_{\mathrm{a}}$ & $1.0 \mathrm{E} 5$ & $\mathrm{~Pa}$ \\
\cline { 2 - 5 } & & & & \\
\hline \multirow{5}{*}{ Explosive (TNT) } & Density & $\rho_{\mathrm{c}}$ & 1630 & $\mathrm{Kg} / \mathrm{m}^{3}$ \\
\cline { 2 - 5 } & Weight & $\mathrm{W}$ & 0.035 & $\mathrm{Kg}$ \\
\cline { 2 - 5 } & & & 0.055 & \\
\cline { 2 - 5 } & & $\rho_{\mathrm{g}}$ & 203.75 & $\mathrm{Kg} / \mathrm{m}^{3}$ \\
\hline \multirow{3}{*}{ Gas bubble } & Density & $\mathrm{P}_{\mathrm{g}}$ & 1.25 & - \\
\cline { 2 - 5 } & Ratio of specific heat & $5.704 \mathrm{E} 7$ & $\mathrm{~Pa}$ \\
\cline { 2 - 5 } & Initial pressure & & \\
\hline
\end{tabular}




\section{RESULTS AND DISCUSSION}

\section{Bubble in Free Field}

Analyzing the complicated cycle of underwater explosion bubbles is challenging, and multiple factors and ideas need to be addressed. Based on BEM findings and experimental results, the results of the FEM were verified (Klaseboer et al., 2005). The CEL results of a series of bubble pulsations and the collapse process were used as a validation method. Based on the BEM findings and experimental results, the results of the FEM were verified (Figs. 1- 8) (Ching-Yu Hsu et al., 2014).

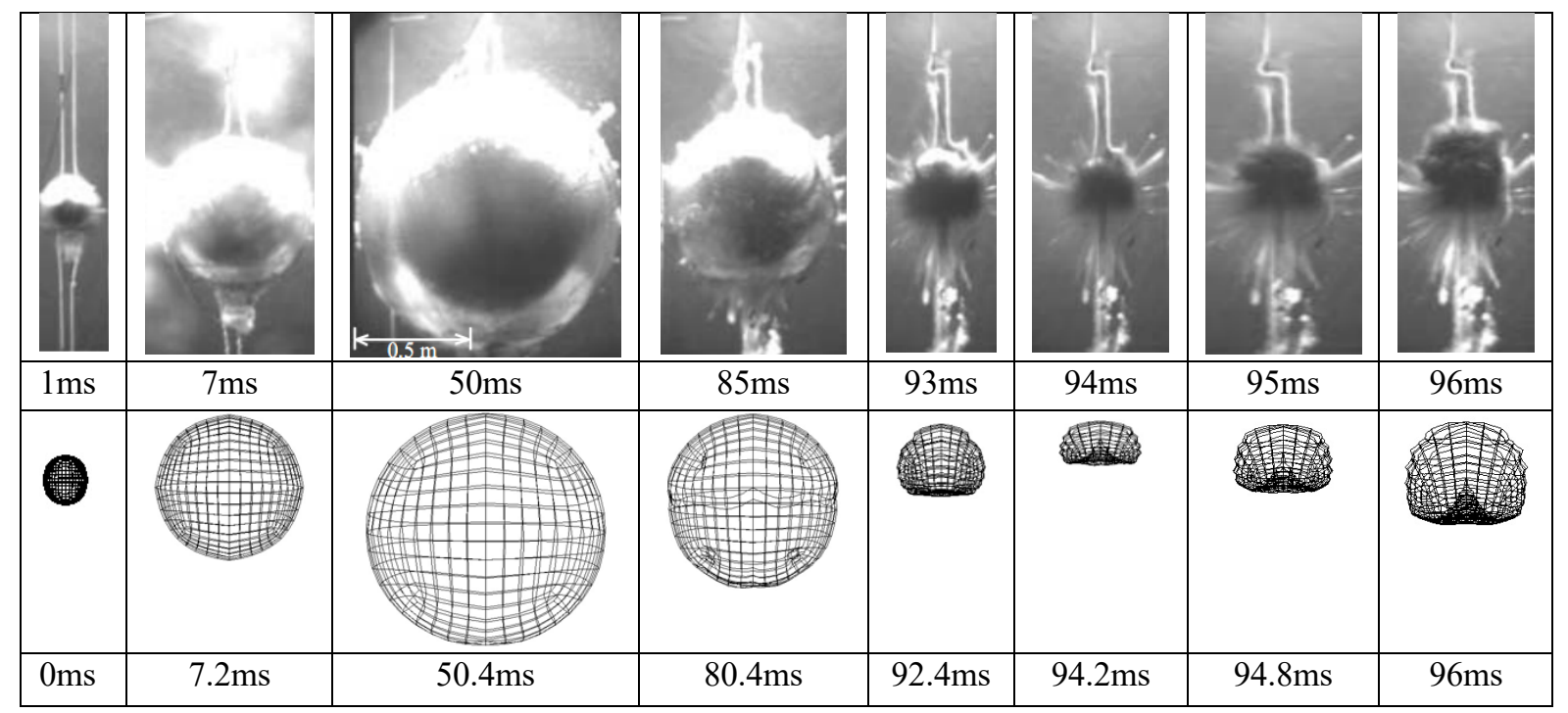

a

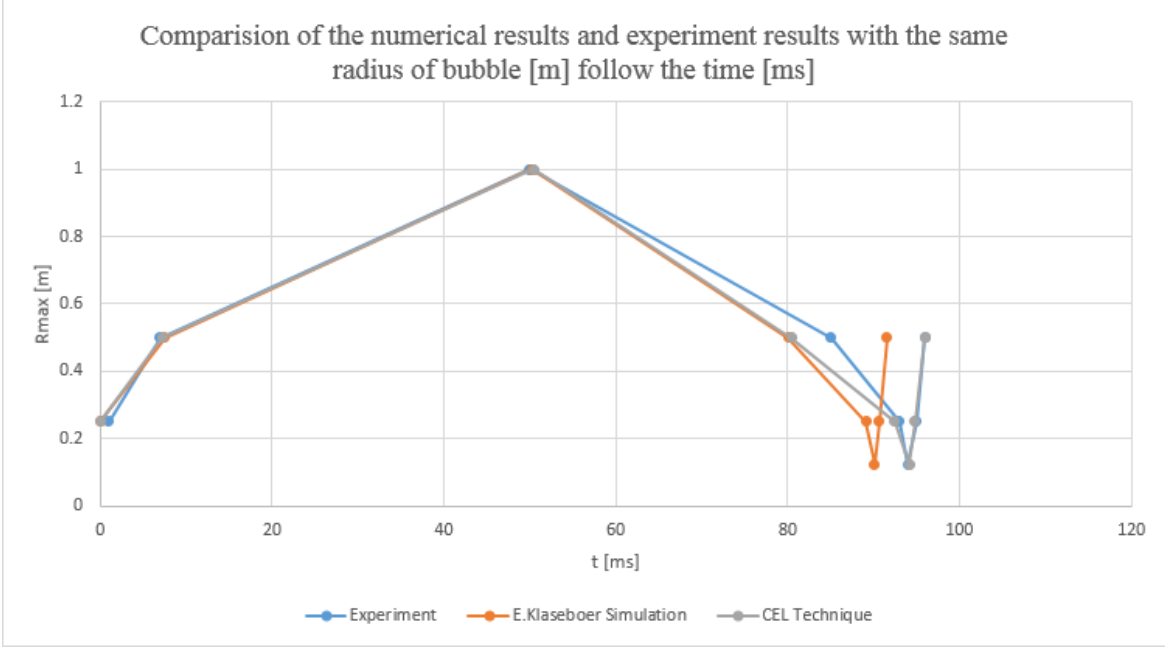

b

Figure 1. The comparison in numerical and experimental results: (a) Klaseboer's experimental and numerical results with similar radius of bubbles and (b) numerical and experimental results in line graph 
(Ching-Yu Hsu etal., 2014).

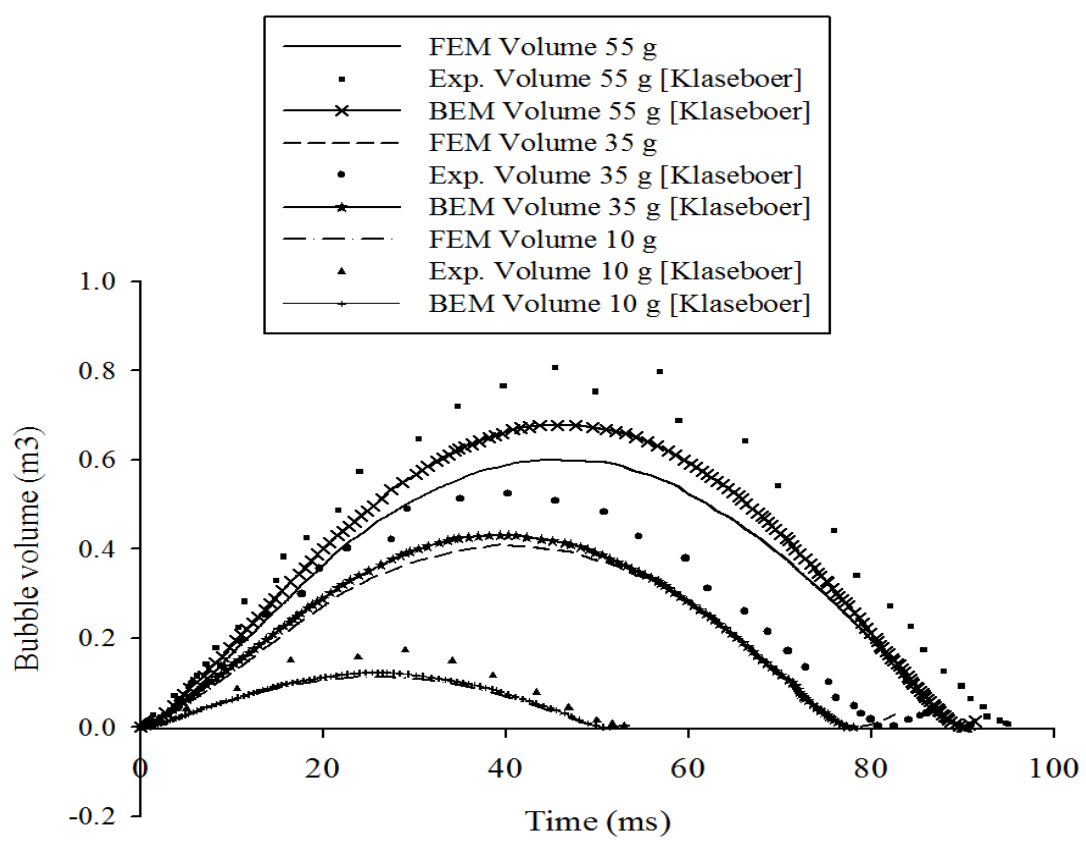

Figure 2. Bubble volume time obtained with different charges ranging from $10 \mathrm{~g}$ to $55 \mathrm{~g}$ in the first circle of bubble oscillation(Ching-YuHsu et al., 2014).

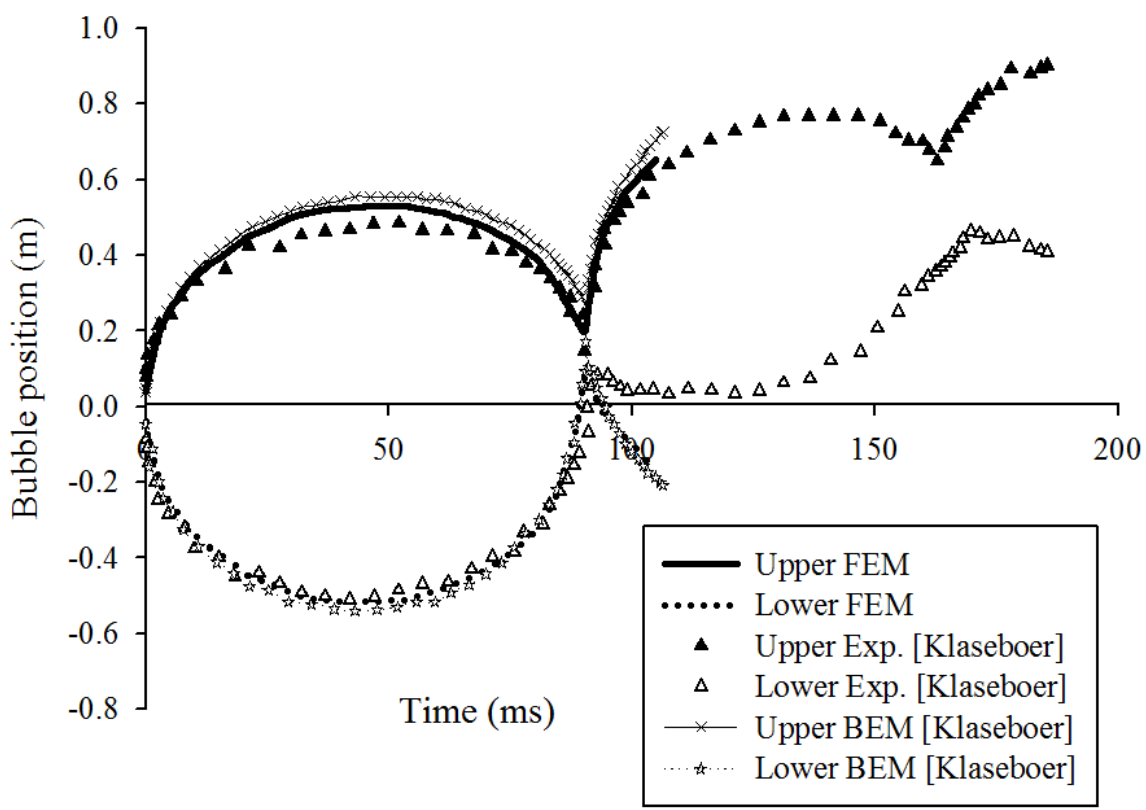

Figure 3. The side of the top and the bottom surface of the bubble (Ching-Yu Hsu et al., 2014). 


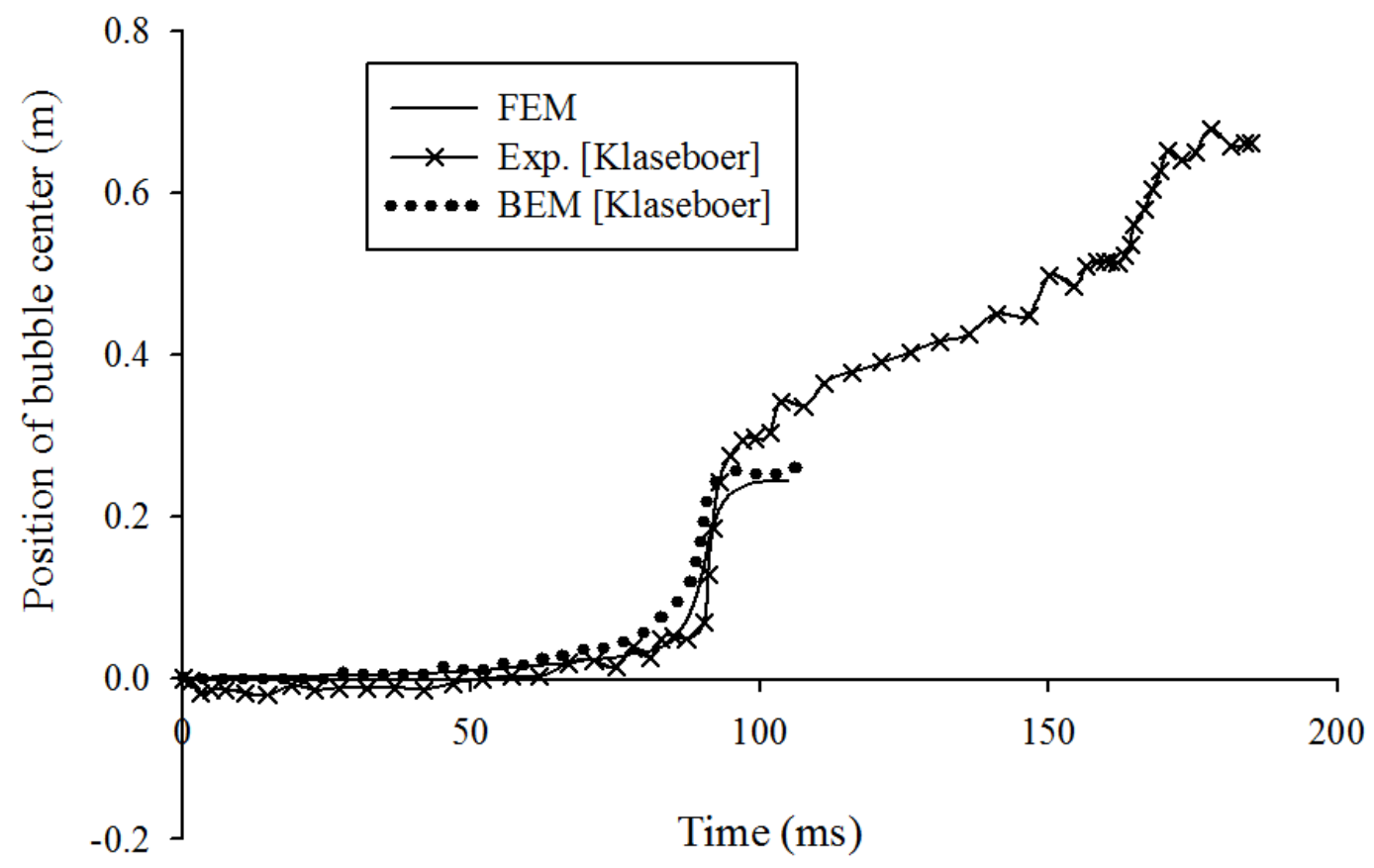

Figure 4. The location of the mass center as a function of time (Ching-Yu Hsu et al., 2014).
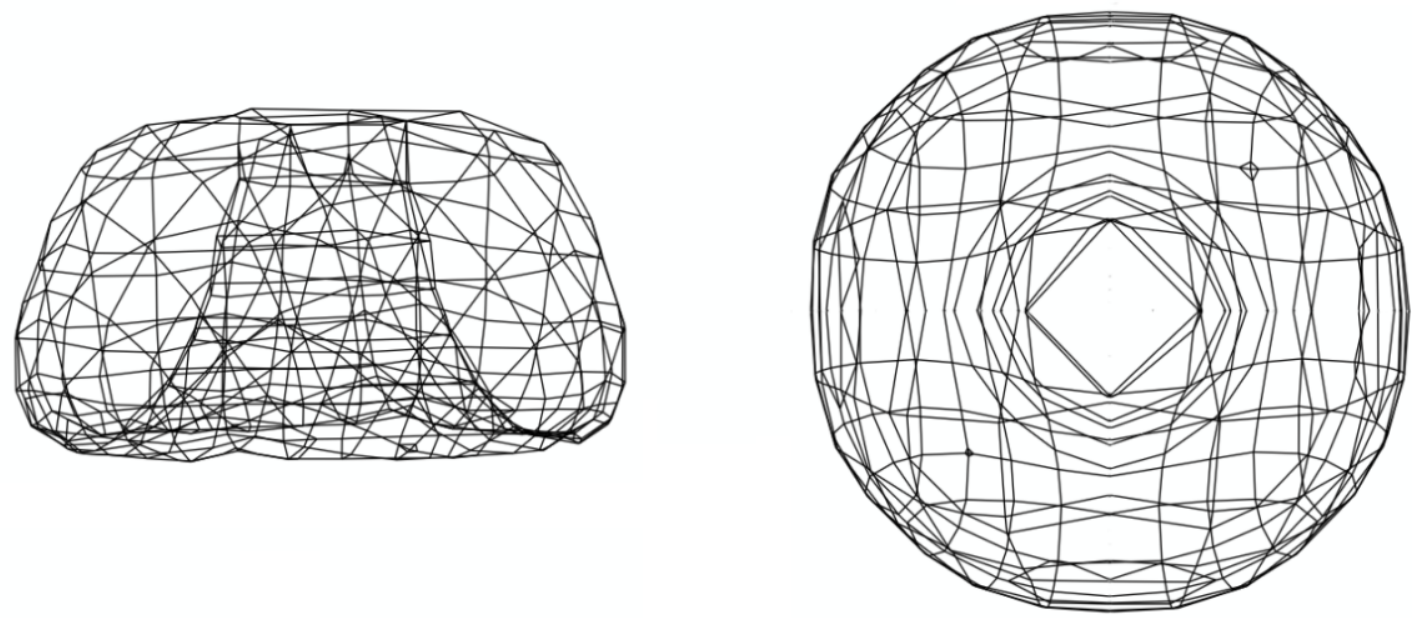

(a)

(b)

Figure 5. The bubble's 3D surface created by a water jet:

(a) side view and (b) top view (Ching-Yu Hsu et al., 2014). 

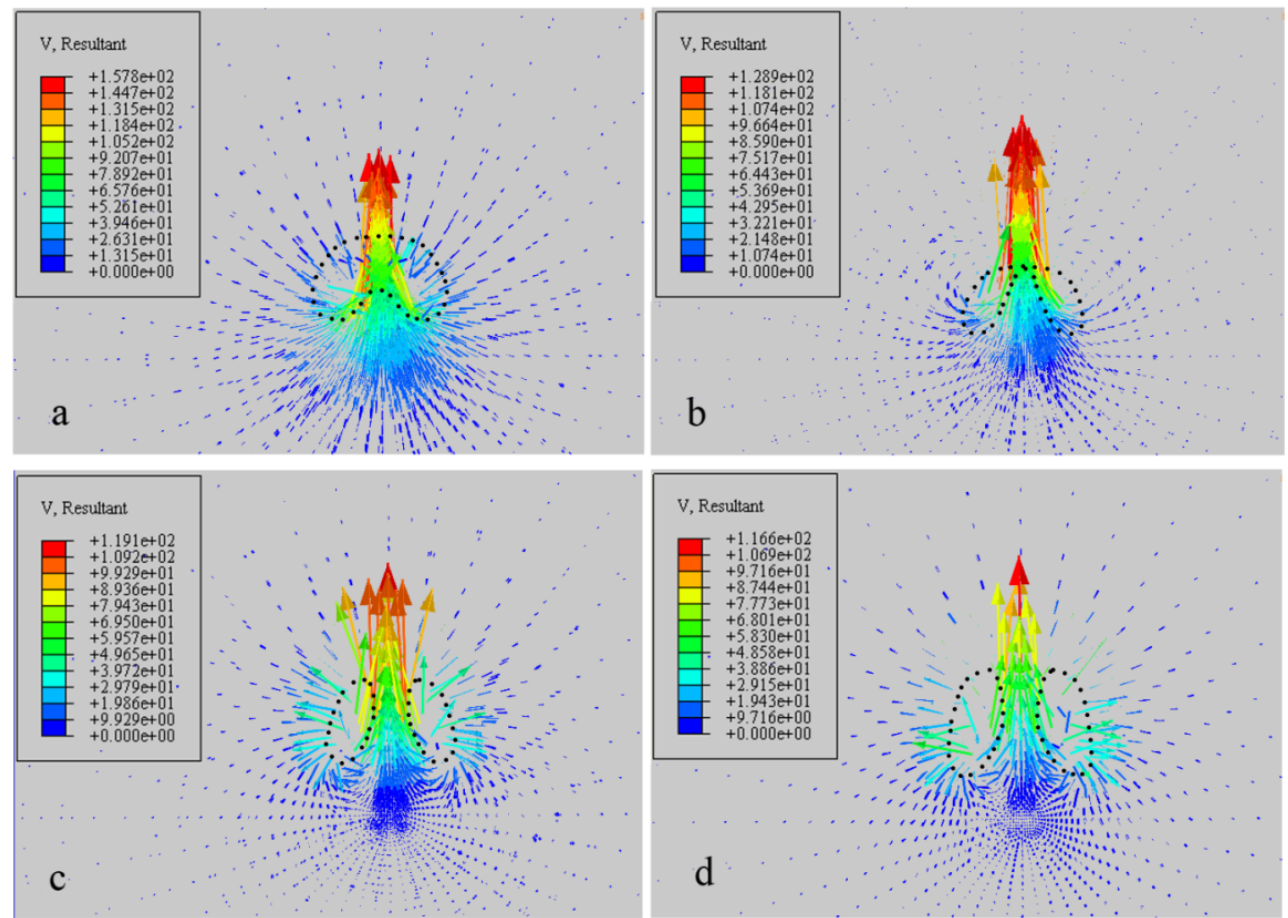

Figure 6. Velocity field of the water surrounding the bubble during the time of breakdown at: (a) $t=90.00 \mathrm{~ms}$, (b) $\mathrm{t}=90.6 \mathrm{~ms}$, (c) $\mathrm{t}=91.12 \mathrm{~ms}$, and (d) $\mathrm{t}=91.18 \mathrm{~ms}$. The dotted line llustrates the bubble boundary (Ching-Yu Hsu et al., 2014).

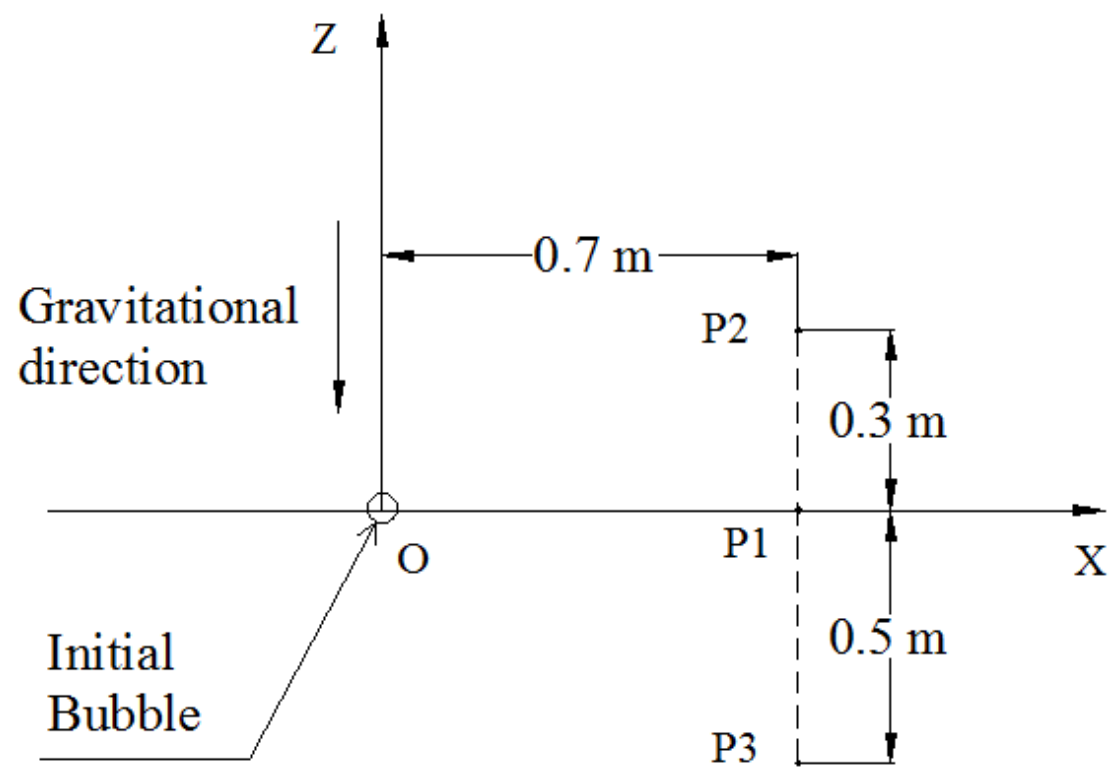

Figure 7. P1, P2, and P3 schematic location (Ching-Yu Hsu et al., 2014). 


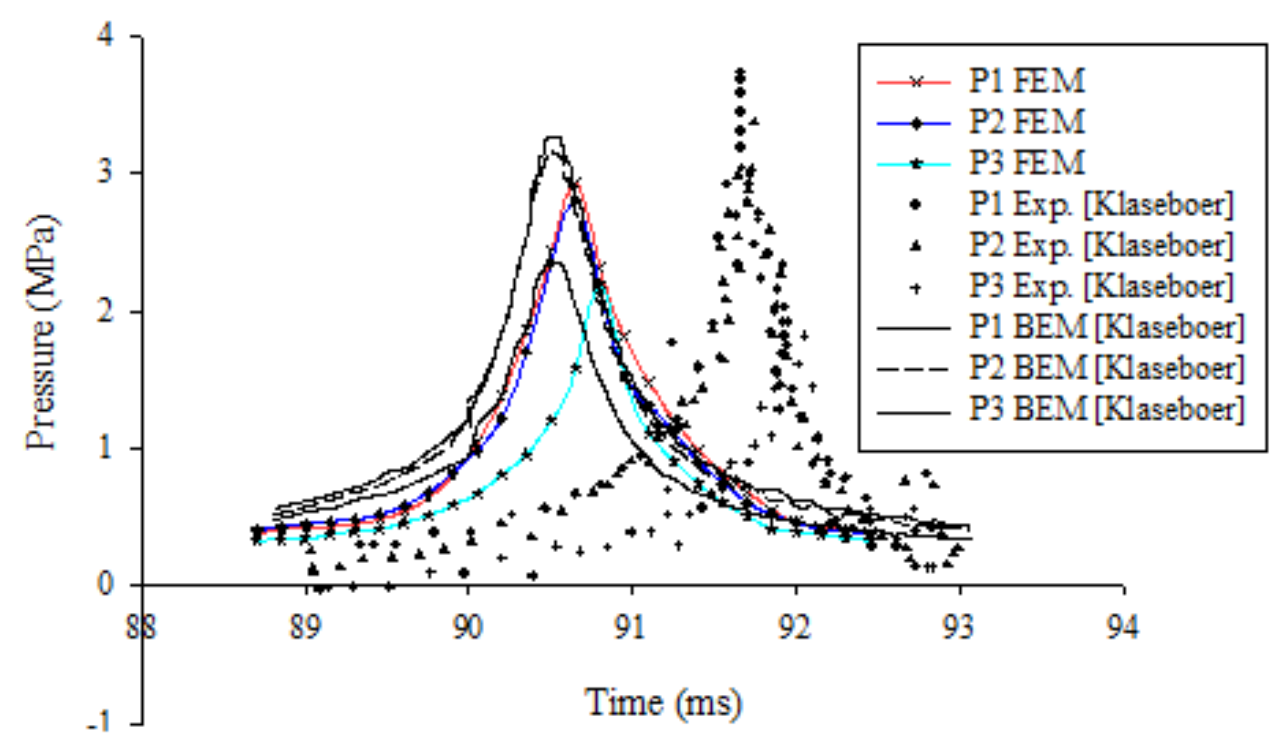

Figure 8. The comparison of pressure pulses around the bubble at different points (Ching-Yu Hsu et al., 2014).

The analysis revealed that the time period profiles were identical to a slight difference between the empirical findings and the experimental results in which the experimental volume was larger than that collected using both the BEM and FEM. This is understandable because hexocire was used to conduct the experiment, whereas, the numerical method was executed using the equivalent mass of TNT. The bubble radius and duration of the first bubble circle obtained using the FEM in all three charge weights were comparable to calculations based on Eq. (7) (Table2).

Table 2. The comparison of bubble radius and duration of first circle between FEM results and empirical results.

\begin{tabular}{|c|c|c|c|c|c|c|}
\hline $\begin{array}{c}\text { Charge } \\
\text { weight }\end{array}$ & $\begin{array}{c}\text { FEM } \\
\text { radius (m) }\end{array}$ & $\begin{array}{c}\text { Empirical } \\
\text { radius (m) }\end{array}$ & $\begin{array}{c}\text { Radius } \\
\text { error (\%) }\end{array}$ & $\begin{array}{c}\text { FEM time } \\
(\mathbf{m s})\end{array}$ & $\begin{array}{c}\text { Empirical } \\
\text { time (ms) }\end{array}$ & $\begin{array}{c}\text { Time error } \\
\mathbf{( \% )}\end{array}$ \\
\hline $10 \mathrm{~g}$ & 0.300 & 0.3068 & 2.21 & 51 & 50.49 & 1.01 \\
\hline $35 \mathrm{~g}$ & 0.460 & 0.4659 & 1.27 & 78.5 & 75.95 & 3.36 \\
\hline $55 \mathrm{~g}$ & 0.523 & 0.5417 & 3.45 & 90.6 & 87.91 & 3.06 \\
\hline
\end{tabular}

In this data, the radius errors of the bubble in the case of $10 \mathrm{~g}, 35 \mathrm{~g}$, and $55 \mathrm{~g}$ were $2.21 \%, 1.27 \%$, and $3.45 \%$, respectively. For further research on bubble migration, this study concentrated on examining the behavior of the bubble produced using $55 \mathrm{~g}$ of TNT. The FEM results were compared to the data reported by Klaseboer et al. (2005). Although the data of these authors was obtained from a bubble below a horizontal plate, the standoff distance between the initial bubble position and the plate was sufficiently large for the bubble to be considered as a bubble in a free field. A unique advantage of the Eulerian procedure suggested in this paper is that valuable information about the process of the bubble collapse can be given using thetool. 


\section{Bubble Near a Rigid Wall}

When the bubble oscillates close to a rigid wall, its direction of migration is influenced by buoyancy forces and Bjerknes forces (Blake, J.R.,1988). This study focused on examining the oscillation of a bubble near a rigid wall through two case studies: a bubble near a vertical wall and a bubble above a horizontal wall. The crucial factor affecting the formation of the water jet, the dimensionless stand-off parameter, was defined as the distance between the initial location of the bubble and the wall $(\mathrm{H})$ scaled according to the maximal bubble radius $\mathrm{R}_{\mathrm{m}}$, which is denoted as $\lambda$ (Blake, J.R.,1988); $\lambda=H / R_{m}$. In this study, the initial depth of the bubble was maintained throughout the simulation, and $\lambda$ was varied to examine the behavior of the bubble and the water jet during the bubble collapse phase. The arrangement of the numerical model was similar to that of the model of the bubble in the free field. The case of a bubble produced using $55 \mathrm{~g}$ of TNT was considered, and the initial depth of the bubble was $3.5 \mathrm{~m}$. The rigid wall, with dimensions $1.5 \mathrm{~m} \times 1.5 \mathrm{~m} \times$ $0.05 \mathrm{~m}$, was relatively large in comparison with the maximum bubble radius; therefore, the bubble essentially behaved like a bubble close to an infinite rigid boundary. All six degrees of freedom of the wall were fixed during the analysis. The enforcement between the Eulerian material flow and the Lagrangianelement of the plate was implemented automatically by the general contact in the ABAQUS software.

\subsection{Bubble in a Nearby Vertical Rigid Wall}

When a bubble oscillates near a vertical rigid wall, it migrates because of the effect of the buoyancy forces and Bjerknes forces produced by the wall. While the buoyancy forces are always directed to the water free surface, the Bjerknes forces are directed toward the wall. Fig. 9 shows the shape of the bubble at different time intervals during the first circle of the bubble at $\lambda=1.1465$.

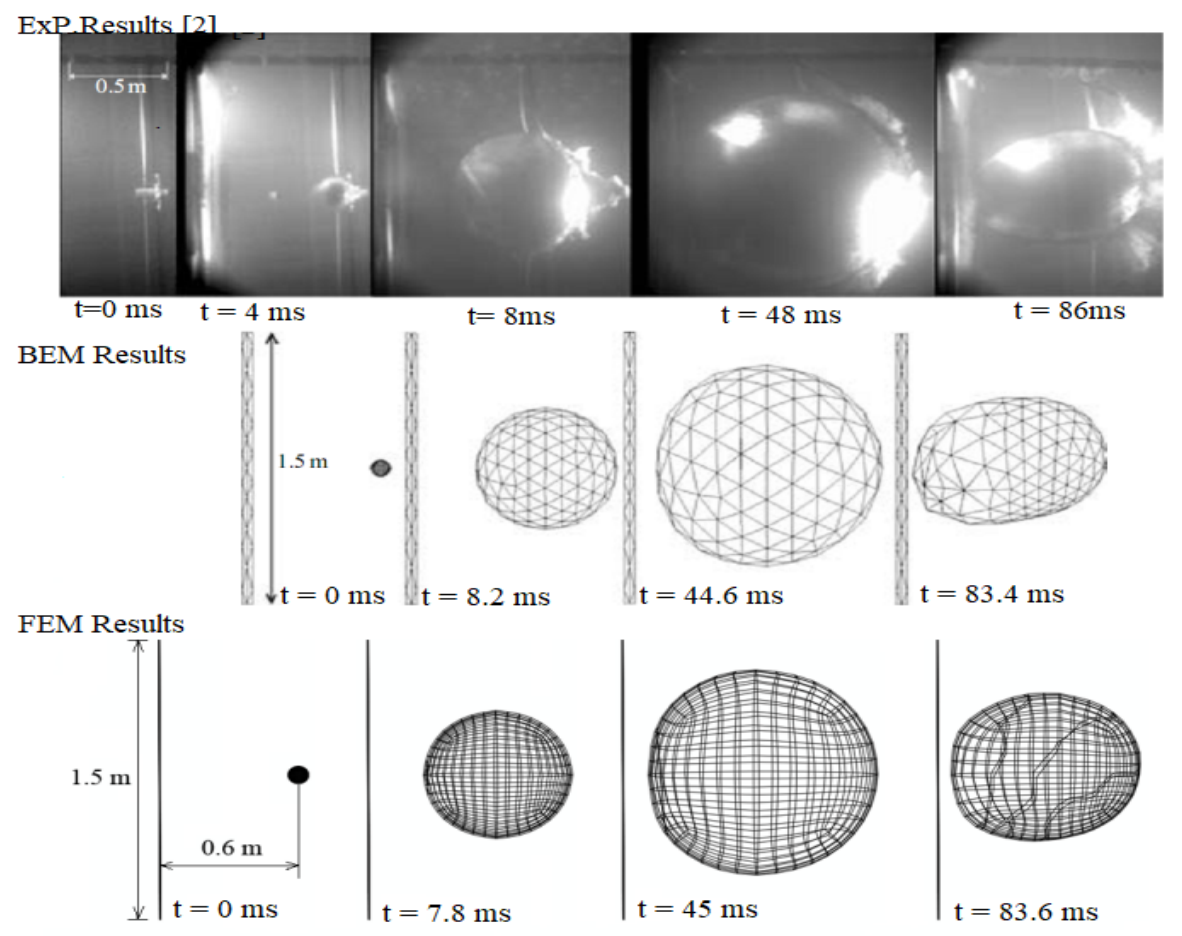

Figure 9. The shape of the bubble near a vertical rigid wall $\mathrm{n}$ comparison among ExP (Klaseboer et al., 2005), BEM, and FEM results at $\lambda=1.1465$. 
The results of the FEM showed good agreement with both the experimental and the BEM results. At this distance, the water jet could be clearly seen in the final stage of the bubble contraction phase; the water jet developed obliquely from the side of the bubble away from the rigid wall and was directed towards it. Fig. 10 shows the velocity field of the water surrounding the bubble during the bubble collapse phase (the wall on the left side of the bubble), in which the high velocity region exhibited the development of a water jet.
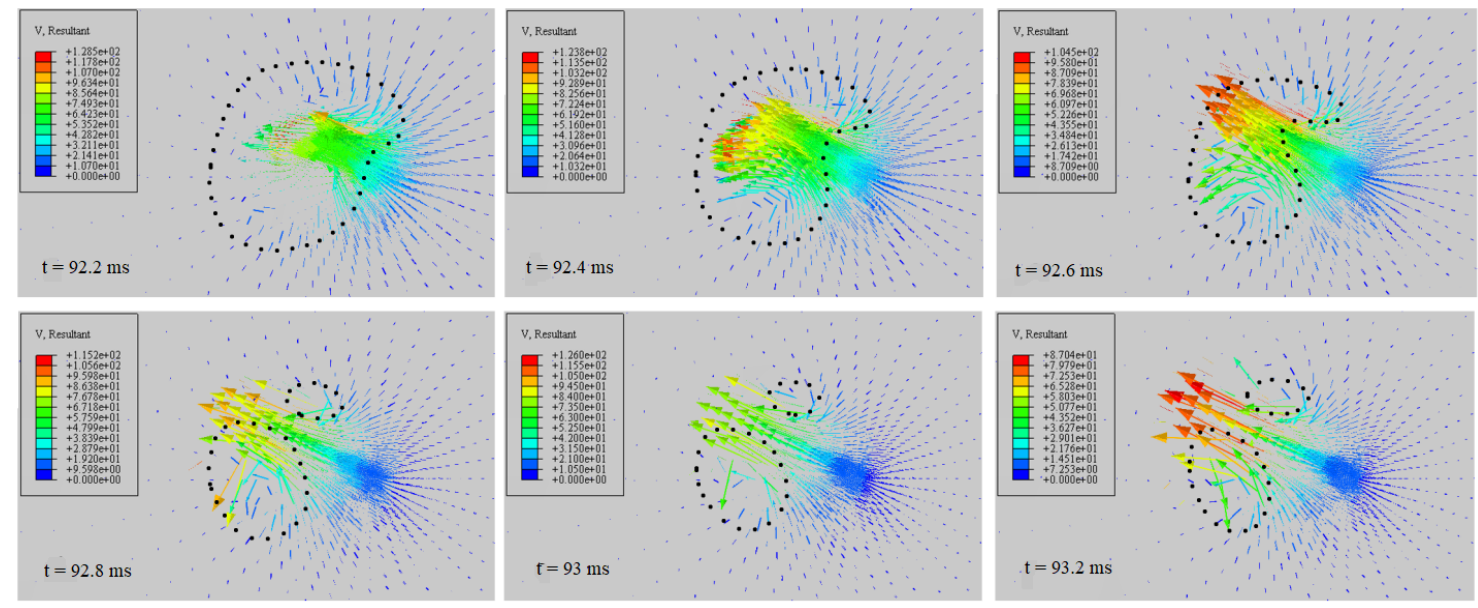

Figure 10. The velocity field of the water surrounding the bubble near a vertical rigid wall at $\lambda=1.1465$, during the collapse phase at different times.

The change in the water jet direction relative to the change of $\lambda$ is shown in Fig. 11. It is clear that, as $\lambda$ increased, the water jet angle (defined as the angle between the direction of the water jet and the horizontal direction) increased. This is understandable because the influence of the wall on the bubble decreased as the standoff distance increased. As $\lambda$ was larger than 2, the effect of the wall on the bubble was almost not realized, and the bubble migrated as it was in a free field. Furthermore, the bubble period increased as $\lambda$ decreased. This suggests that the attraction of the structure prolonged the expansion and contraction time of the bubble.
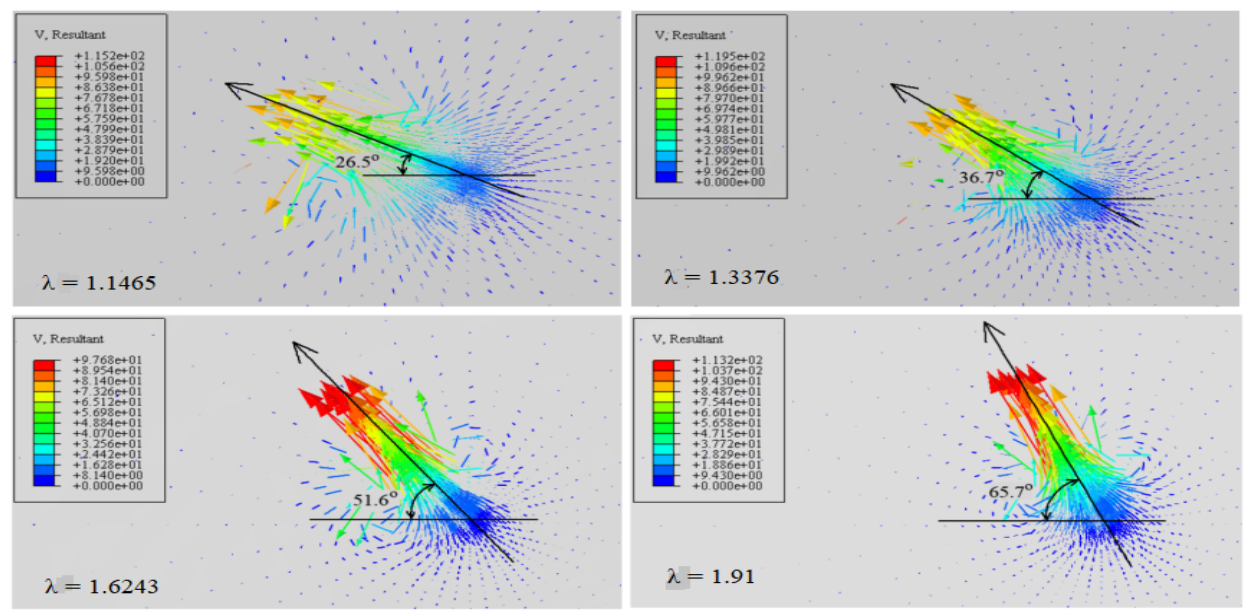

Figure 11. The change $n$ water jet velocity angle of the bubble near a vertical rigid wall at a double connected bubble at different standoff distances: $\lambda=1.1465 ; \lambda=1.3376 ; \lambda=1.6243$, and $\lambda=1.91$, respectively. 


\subsection{Bubble above a Horizontal Rigid Wall}

When the bubble oscillates above a horizontal rigid wall, the buoyancy forces and Bjerknes forces act in opposite directions (Bjerknes forces are defined as the translational forces placed in pulsating air bubbles in an acoustic field) (Blake,J.R.,1988).

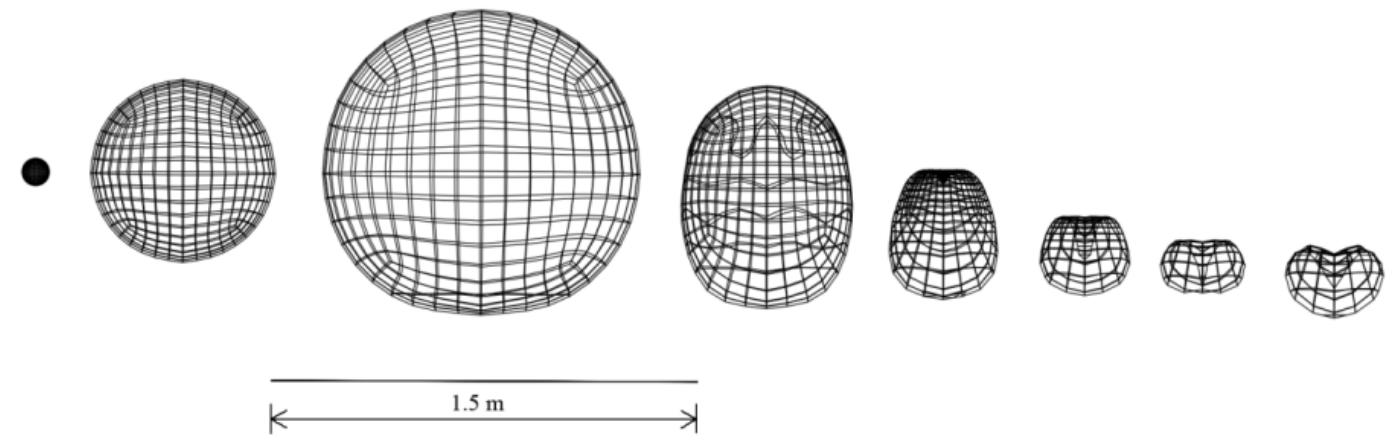

Figure 12. The shape of bubble above a horizontal rigid wall at $\lambda=1.1465$. FEM results at: $t=0,7.6,48.2,79.2,86.8$, $89.64,90.8$, and $91.6 \mathrm{~ms}$.

The migration of the bubble and the development of the water jet depend strongly on the standoff distance between the initial position of the bubble and the wall. Fig. 12 shows the shape of the bubble above the rigid wall at different time intervals when $\lambda=1.1465$ and the water jet that developed downward. Fig. 13 illustrates the displacement of the bubble centroid because of the change in $\lambda$. In all cases, the buoyancy forces dominated during the expansion phase, and the bubble migrated slightly upwards. However, the effects of the Bjerknes forces were clearer in the collapse phase of the bubble located close to the wall. When $\lambda$ was less than 1.815 , the Bjerknes forces were dominant; therefore, the bubble migrated toward the wall and the water jet developed downward directly toward the wall (Fig. 12). As $\lambda$ increased, the attraction of the wall to the bubble decreased. The simulation results show that, when $\lambda$ was 1.815 , the position of the bubble centroid barely changed after the first bubble circle. This phenomenon is known as the null Kelvin impulse state of the bubble, in which the buoyancy forces and Bjerknes forces are nearly equal (Blake, J.R., 1988).

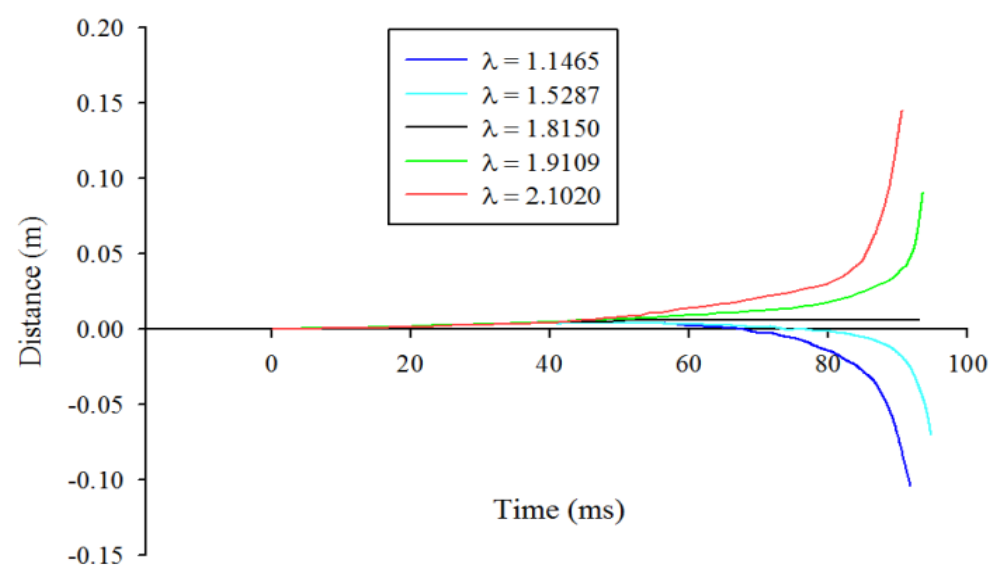

Figure 13. The displacement of the bubble centroid in the case of a bubble above a horizontal rigid wall at different standoff distances: $\lambda=1.1465, \lambda=1.5287, \lambda=1.815, \lambda=1.91$ and $\lambda=2.102$ 
When $\lambda$ was greater than 1.815 , the buoyancy forces predominated; therefore, the bubble moved upward, and the water jet was directed to the water free surface. When $\lambda$ was greater than 2 , the effect of the wall almost vanished. Bubble migration was similar to that in the free domain, as shown in Fig. 13.

\section{CONCLUSION}

The CEL technique in the ABAQUS/Explicit 6.11 code presented in this paper was applied to reproduce an UNDEX bubble in an experiment. This study shows the potential application of a code for finite element analysis to quantitatively model the UNDEX bubble, namely ABAQUS 6.11. Future studies will expand the applicability of the findings to real conditions, such as the relationships between UNDEX bubbles and structures made up of various materials. In order to obtain more accurate results, care should be taken to consider the effect of the mesh solution, fluid compressibility, and heat exchange. These studies would be difficult, requiring deep theoretical material, but would also deliver useful and convincing findings. In addition, the implications of water jet modes need to be addressed, and the results of this study will be a prospective tool of testing for future UNDEX research projects.

\section{REFERENCES}

Cole, R.H., 1948. Underwater explosions, Princeton University Press, Princeton, NJ.

Klaseboer, E., Hung, K.C., Wang, C., Khoo, B.C., Boyce, P., Debono, S., Charlier, H., 2005. Experimental and numerical investigation of the dynamics of an underwater explosion bubble near a resilient/rigid structure. J. of Fluid Mech. 537, 387-413.

Brett, J.M., Yiannakopoulos, G., Schaaf, P. J., 2000. Time-resolved measurement of the deformation of submerged cylinders subjected to loading from nearby explosion. International Journal of Impact Engineering. 24, 875-890.

Blake, J.R., Taib, B.B., Doherty, G., 1987. Transient cavities near boundaries. Part 2. Free surface. J. Fluid Mech. 181, 197-212.

Zhang, Y.L., Yeo, K.S., Khoo, B.C., Chong, W.K., 1998. Three-dimensional computation of bubbles near a free surface. J. of Compt. Phys. 146, 105-123.

Wang, C., Khoo, B.C., Yeo, K.S., 2.B., Doherty, G., 1987. Transient cavities near boundaries. Part 2. Free surface. J. Fluid Mech. 181, 197-212.

Jin Qiankun, Ding Gangyi, 2011. A finite element analysis of ship sections subjected to underwater explosion. International Journal of Impact Engineering, vol 38, pages 558-566.

Hans U. Mair, 1995. Hydro-code methodologies for underwater explosion structure/medium interaction. Proceeding of the $66^{\text {th }}$ Shock and Vibration Symposium, Volume II, SAVIAC, 227- 248.

Jan Erik Olsen, Paal Skjetne, 2016. Modelling of underwater bubble plumes and gas dissolution with an Eulerian-Lagrangian CFD model. Applied Ocean Research, Vol.59, PP.A3-200.

David Cherney, Tom Denton, Rohit Thomas and Andrew Waldron, 2013. Linear Algebra, Edited by Katrina Glaeser and Travis Scrimshaw. First Edition, Davis California.

ABAQUS Analysis User's Manual and ABAQUS Theory Manual, 2011. Version 6.11.1 Dassault Systèmes, RI, USA.

Ching-Yu Hsu, Cho-Chung Liang, Anh Tu Nguyen, 2014. A numerical study on the underwater explosion bubble pulsation and the collapse process. Ocean Engineering 81 (2014) 29-38.

Blake, J.R., 1988. The Kelvin impulse: Application to cavitation bubble dynamics. J. Austral. Math. Soc. Ser. B $30,127-146$. 